\title{
Synchronized necrotic death of attached hepatocytes mediated via gap junctions
}

SUBJECT AREAS:

CELLULAR IMAGING

CELL DEATH

Received

25 February 2014

Accepted

14 May 2014

Published

4 June 2014

Correspondence and requests for materials should be addressed to Y.T. (tsujimoł@gene. med.osaka-u.ac.jp)

\author{
Chieko Saito $^{1,2}$, Koei Shinzawa ${ }^{2}$ \& Yoshihide Tsujimoto ${ }^{2}$
}

'Japan Society for the Promotion of Science (JSPS), '2Department of Medical Genetics, Osaka University Medical School, Osaka, Japan.

Extensive studies have unveiled the intracellular molecular signaling pathways of cell death. To better understand cell death in tissues, it is important to investigate the influence of neighboring cells on the response to death stimuli. By time-lapse microscopy, we found that cells in couplets (two hepatocytes attached to each other) died independently when stimulated with anti-Fas antibody and staurosporine, whereas acetaminophen (APAP) and aryl alcohol caused synchronized cell death although its timing varied among different couplets. Synchronized death of couplets was not caused by APAP when hepatocytes were deficient in both Connexin 26 and Connexin 32 , indicating a crucial role of gap junctions in the synchronized death process. We also demonstrated that APAP-sensitive male hepatocytes were protected by attachment to APAP-insensitive female hepatocytes, with this protection being dependent on gap junctions. These findings indicate that APAP-induced and aryl alcohol-induced necrotic death of hepatocytes is modulated by attached neighboring cells via gap junctions.

C ell death has long aroused the interest of various scientific communities, because it not only plays a physiological role in morphogenesis during development and in the turnover of cells in various tissues, but also has a pathological role in many diseases. Extensive studies performed over the last decade have unveiled the mechanisms of apoptosis, as well as some forms of non-apoptotic cell death such as programmed necrosis/necroptosis.

For detailed analysis of cell death mechanisms, cultured cells have been used successfully, but better understanding of cell death in tissues should require investigation of intercellular communication, because each cell in a tissue is affected by its neighbors via factors that are secreted into the microenvironment as well as by direct cellto-cell communication via gap junctions or other methods. Studies performed during last decade on cell death in tissues have uncovered some interesting phenomena. One of these is compensatory proliferation, which was originally discovered in Drosophila, i.e., apoptotic death of a cell in a tissue enhances the proliferation of neighboring cells ${ }^{1}$. Another is the possible role of gap junctions in cell death. For example, it was reported that streptozotocin and alloxan induce selective and massive apoptotic death of pancreatic beta cells, which is prevented by connexin $(\mathrm{Cx}) 36$, a constituent of gap junctions between beta cells in the pancreatic islets of mice ${ }^{2}$. In contrast, hepatotoxicity of drugs, such as D-galactosamine, carbon tetrachloride, and acetaminophen (APAP), was reported to be reduced in rats with a dominant-negative mutation of $\mathrm{Cx} 32^{3,4}$.

To better understand cell death in tissues, we chose the mouse liver and hepatocytes as a model, because of our interest in both mammalian cell death mechanisms and also in developing new therapeutic strategies for liver diseases. Hepatocytes are known to have sites of tight intercellular adhesion where gap junctions form. Gap junctions are channels that are typically found in clusters ranging from 10 to 10,000 called plaques on the cell membrane $^{5}$. A gap junction is composed of two opposing hemichannels that consist of six connexin $(\mathrm{Cx})$ proteins, with Cx32 and Cx26 being major constituents of gap junctions in hepatocytes ${ }^{5}$. Gap junctions allow transfer of molecules smaller than $\sim 1 \mathrm{kDa}$, such as ions, metabolites, reactive oxygen species (ROS), and second messengers, to the adjacent cells ${ }^{6-8}$, and are known to be involved in various biological processes, such as cell differentiation, growth, and death ${ }^{5}$.

We studied the death of hepatocytes attached to other hepatocytes and found that treatment with APAP or aryl alcohol caused synchronized necrotic death of attached hepatocytes, which was mediated via gap junctions.

\section{Results}

Acetaminophen induces synchronized necrotic death of attached hepatocytes. To investigate whether death of a cell in response to external death stimuli is influenced by the surrounding cells, we employed primary cultured mouse hepatocytes and focused on attached hepatocytes. As death inducers, acetaminophen (APAP) and an anti- 
Fas antibody with cycloheximide (CHX) were used, which induce necrotic and apoptotic cell death of primary cultured hepatocytes, respectively. After addition of the death stimulus, attached hepatocytes were observed by time-lapse microscopy. To assess anti-Fas antibody-induced apoptosis, the timing of cell death was monitored by detachment of cells from the culture dish. On the other hand, the timing of APAP-induced necrotic death was determined by loss of fluorescence of tetramethylrhodamine methyl ester (TMRM), which accumulates in mitochondria with an intact membrane potential. Most hepatocytes became PIpositive within $10 \mathrm{~min}$ after loss of the mitochondrial membrane potential as assessed by TMRM fluorescence (data not shown). As shown in Fig. 1c-f, attached hepatocytes died simultaneously in a group after stimulation with APAP, although the timing of death differed between independent groups of hepatocytes. In contrast, attached hepatocytes died independently in response to anti-Fas antibody treatment (Fig. 1a, b). To further investigate APAPinduced death of attached hepatocytes, we focused on couplets, i.e., two hepatocytes attached to each other. To quantitatively assess whether hepatocytes in couplets underwent synchronized death in the presence of APAP, we conducted an analysis of the timing of death for each hepatocyte in the couplets. As shown in Fig. 1h, cells in a large proportion of couplets showed almost completely synchronized death $(\mathrm{r}=0.883)$, although the timing of death varied among couplets, and only a small fraction of couplets showed independent hepatocyte death. In contrast, each hepatocyte in the couplets died individually after anti-Fas antibody stimulation ( $r=0.339$ ) (Fig. 1g). Synchronized death of hepatocytes was not specific to APAP, because similar synchronization of necrotic death was observed after exposure to allyl alcohol, while staurosporine induced independent necrotic death of hepatocytes in couplets (Supplementary Fig. S1). These results demonstrated that some (but not all) death stimuli, such as APAP and allyl alcohol, induce synchronized death of attached hepatocytes.

It has been reported that APAP and allyl alcohol cause hepatocyte death in the centrilobular and periportal regions, respectively ${ }^{9,10}$. Therefore, there was a possibility that two attached hepatocytes in culture had already been attached to each other in the liver and had inherited similar properties, which could result in synchronized cell death. To test this possibility, the timing of APAP-induced cell death was studied in couplets that were only attached together after the start of culture, which were called post-couplets. Hepatocytes were first stained with a dye and unstained hepatocytes were added to the same dish after washing off the free dye. Then we analyzed couplets formed by unstained and stained hepatocytes. As shown in Fig. 2b, hepatocytes in post-couplets underwent APAP-induced synchronized death, like the couplets prepared as described in Fig. 1, which included both couplets attached in the liver and those attached in culture (Fig. 2a). These results suggested an important role of cell-tocell communication in the synchronization of cell death in couplets.

Synchronized death caused by APAP is attributable to gap junctions. There are two methods of cell-to-cell communication, with one involving paracrine secretion of soluble factors and the other being intercellular transfer of factors via gap junctions. As synchronized cell death was only observed for attached hepatocytes and not unattached hepatocytes in close proximity (data not shown), we examined the possible role of gap junctions in synchronized death caused by APAP. Gap junctions in hepatocytes are formed by heteromeric and homomeric hexamers of $\mathrm{Cx} 26$ and $\mathrm{Cx} 32$. To eliminate gap junctions from hepatocytes, we obtained hepatocytes from $\mathrm{Cx} 32 \mathrm{KO}$ mice and treated them with $\mathrm{Cx} 26$ siRNA since mice deficient for Cx26 undergo embryonic death ${ }^{11}$. Cx26 expression was greatly diminished by treatment with Cx26 siRNA for $13 \mathrm{~h}$ in both hepatocytes from WT and Cx32 KO mice, as shown by western blotting (Fig. 3a). The validity of the Cx26 signal detected by western blotting was confirmed by using Cx26overexpressing hepatocytes as a positive control (data not shown). As shown in Fig. 3b, depletion of Cx26 and Cx32 from hepatocytes did not affect total cell viability at $24 \mathrm{~h}$ after APAP treatment, as assessed by the CTB assay, suggesting that gap junctions did not influence the viability of primary cultured hepatocytes. In contrast to WT hepatocyte couplets that showed synchronized death after APAP stimulation $(\mathrm{r}=0.935)$ (Fig. 4a), synchronized cell death was not observed with couplets of hepatocytes deficient in both $\mathrm{Cx} 26$ and $\mathrm{Cx} 32(\mathrm{r}=0.240)$ (Fig. 4d). Elimination of either $\mathrm{Cx} 26$ or $\mathrm{Cx} 32$ reduced the extent of synchronization of cell death $(\mathrm{r}=$ $0.539, \mathrm{r}=0.579$, respectively) (Fig. $4 \mathrm{~b}, \mathrm{c}$ ). Similar results were also obtained when a different siRNA for Cx26 (Cx26 siRNA1) was used, excluding the possibility of an off-target effect (Supplementary Fig. S2). Taken together, these findings indicated that synchronized cell death after exposure to APAP depends on functional gap junctions.

APAP-sensitive male hepatocytes are protected from APAPinduced death by APAP-resistant female hepatocytes via gap junction-dependent cell-to-cell communication. To further study the role of cell-to-cell communication via gap junctions in APAPinduced cell death, we formed couplets of hepatocytes with differing sensitivity to APAP. It has been reported that male mice are more sensitive to APAP than female mice independent of APAP metabolism $^{12-14}$. Therefore, we prepared couplets that contained male and female hepatocytes, as described in Fig. 5a. We prepared two dishes; hepatocytes from male WT mice were seeded in the first dish and stained, after which female WT hepatocytes were added with control siRNA. Hepatocytes from male Cx32 KO mice were seeded in the second dish and stained, after which female $\mathrm{Cx} 32 \mathrm{KO}$ hepatocytes were added with Cx26 siRNA. Knockdown of Cx26 was confirmed by western blotting (Supplementary Fig. S3). Formation of functional gap junctions between male and female hepatocytes in the couplets was demonstrated by the Calcein AM dye transfer assay (Supplementary Fig. S4). We then analyzed 20 randomly selected couplets each of male-male, female-female, and male-female hepatocytes from each dish. Confirming the previous observation, female couplets from WT and $\mathrm{Cx} 32 \mathrm{KO}$ mice were more resistant to APAP than male hepatocytes $(89 \pm 3 \%$, and $93 \pm 5 \%$ viability of WT and Cx32 KO female hepatocytes, respectively, versus $45 \pm 9 \%$ and $36 \pm 8 \%$ viability of WT and $\mathrm{Cx} 32 \mathrm{KO}$ male hepatocytes, respectively) (Fig. 5b, c). Death of these hepatocytes occurred in a synchronized manner (data not shown).

In approximately $80 \%$ of WT male-female couplets, both male and female hepatocytes survived (Fig. 5d), indicating that many APAPsensitive male hepatocytes were protected from APAP-induced death by APAP-resistant female hepatocytes. To determine whether the protection of male hepatocytes by female hepatocytes was dependent on gap junctions, we examined male-female couplets derived from $\mathrm{Cx} 32 \mathrm{KO}$ mice and then with $\mathrm{Cx} 26$ siRNA. As shown in Fig. 5d, in the absence of gap junctions, male hepatocytes were not protected from APAP-induced death by viable female hepatocytes. These results indicated that APAP-sensitive male hepatocytes were protected from APAP-induced death by APAP-resistant female hepatocytes through intercellular communication via gap junctions.

\section{Discussion}

During the past decade, the physiological and pathological roles of cell death via cell-to-cell communication have been studied. In this study, we first demonstrated an interesting phenomenon of synchronized necrotic cell death mediated though intercellular communication via gap junctions. By using time-lapse microscopy, we showed that APAP and aryl alcohol induced synchronized necrotic death of attached hepatocytes, although the timing of death varied among different groups of hepatocytes. In contrast, anti-Fas antibody and staurosporine induced independent death of attached hepatocytes. 
(a)

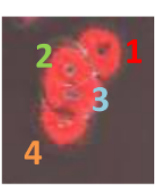

0

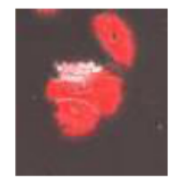

600

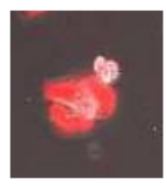

890

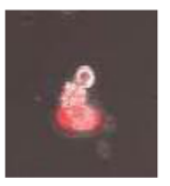

1220

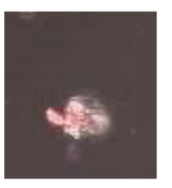

1420

(min)

(b)

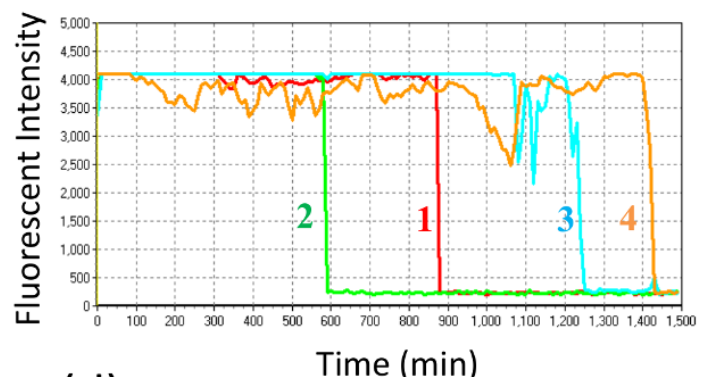

(d)
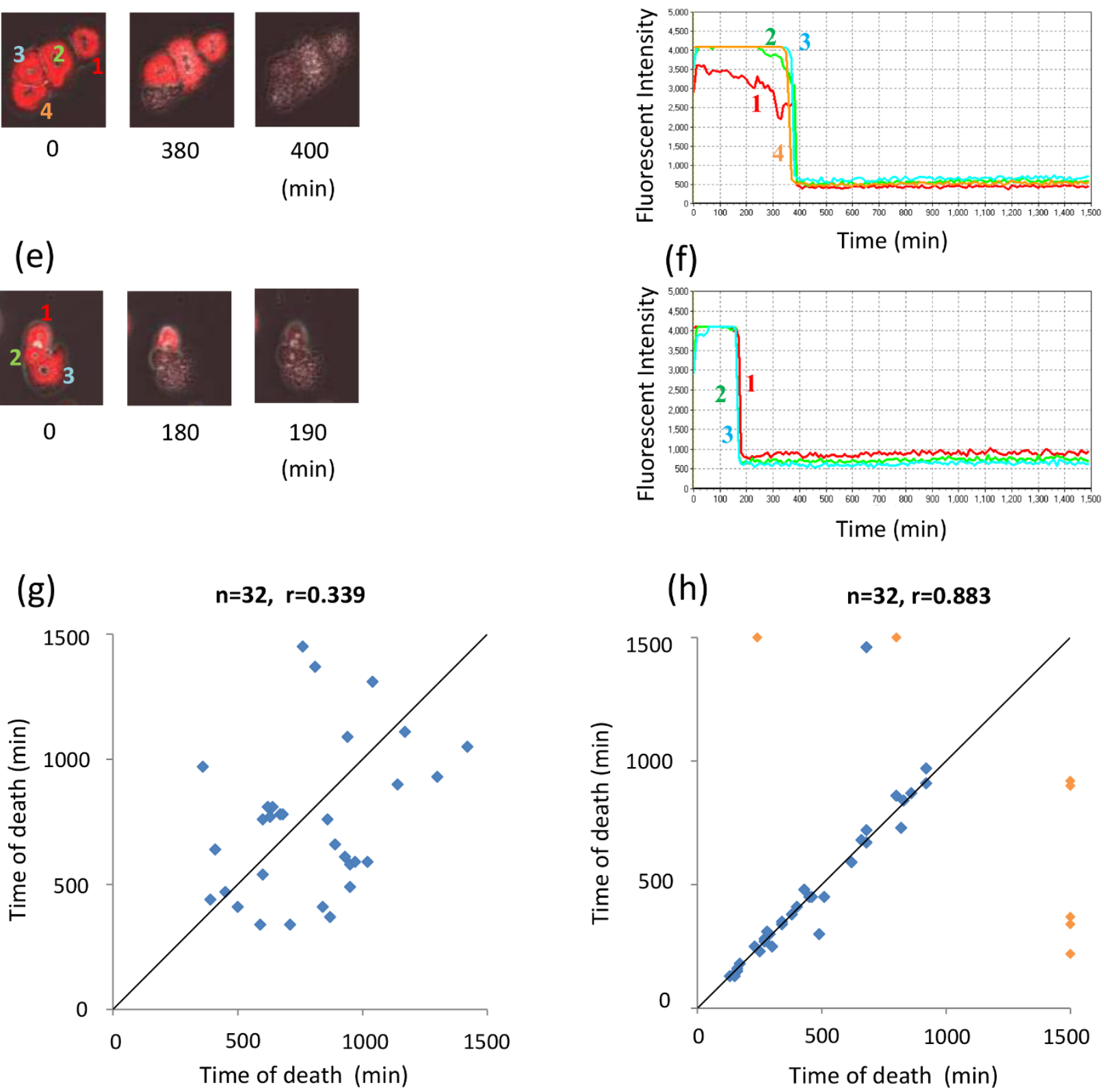

(f)

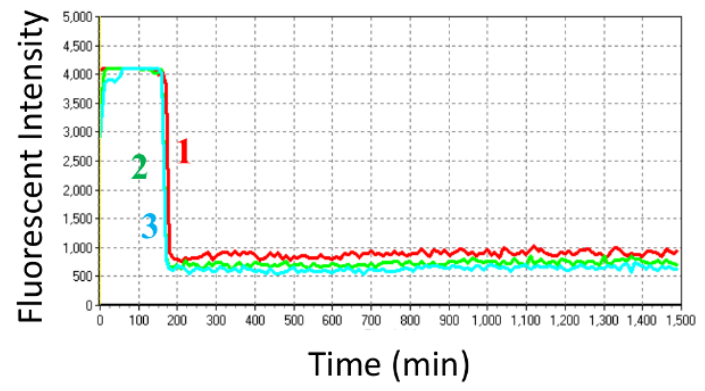

(h)

$n=32, r=0.883$
190

(min)
Time (min)
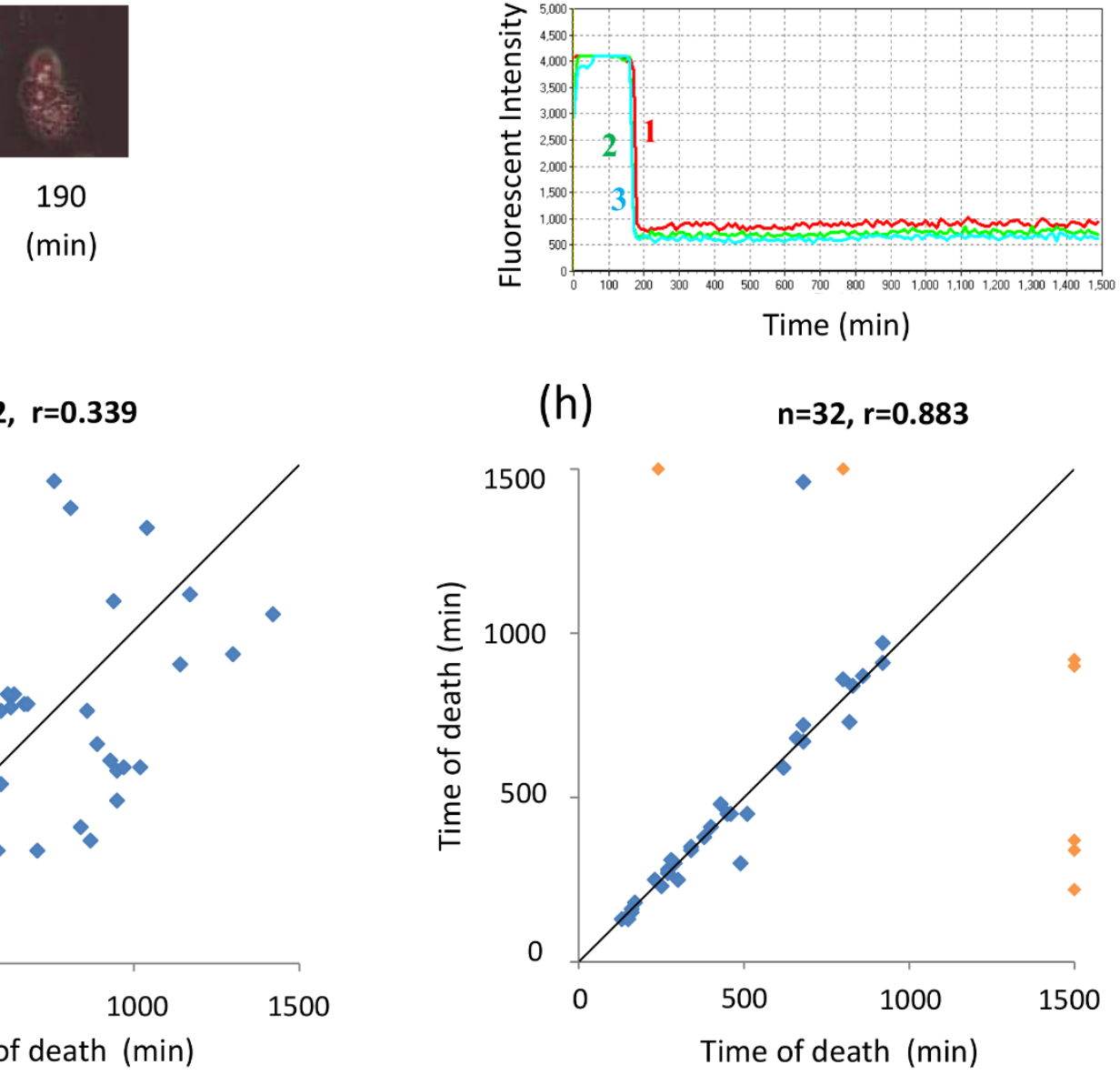

Figure 1 APAP, but not anti-Fas antibody, causes synchronized death of attached hepatocytes. Primary cultured murine hepatocytes were exposed to $0.5 \mu \mathrm{g} / \mathrm{ml}$ anti-Fas antibody with $50 \mu \mathrm{g} / \mathrm{ml} \mathrm{CHX} \mathrm{(a,} \mathrm{b,} \mathrm{g)} \mathrm{or} \mathrm{to} 10 \mathrm{mM}$ APAP (c-f, h). TMRM was used as an indicator of cell death. However, for Fasmediated death, detachment of cells from the dish was regarded as evidence of death. Images were obtained every $10 \mathrm{~min}$ until $1,500 \mathrm{~min}$ by time-lapse microscopy. Representative images obtained at the start of exposure to stimuli $(0 \mathrm{~min})$ and at the indicated time points are shown (a, c, e). Fluorescence intensity was measured over time for numbered cells (a, c, e) and shown in (b, d, f). Cells in (c, e) were two different groups of hepatocytes in the same dish. For cell couplets (two attached hepatocytes), the time of death of one cell in the couplet was plotted on the X axis and that of the other cell was plotted on the $\mathrm{Y}$ axis $(\mathrm{g}, \mathrm{h}$ ). Blue dots show couplets in which both cells died within 1,500 min and orange dots indicate couplets in which at least one of the hepatocytes did not die within 1,500 min of exposure to APAP. $\mathrm{n}$ is the number of couplets, $\mathrm{r}$ is the correlation coefficient (orange dots were excluded). 
(a)

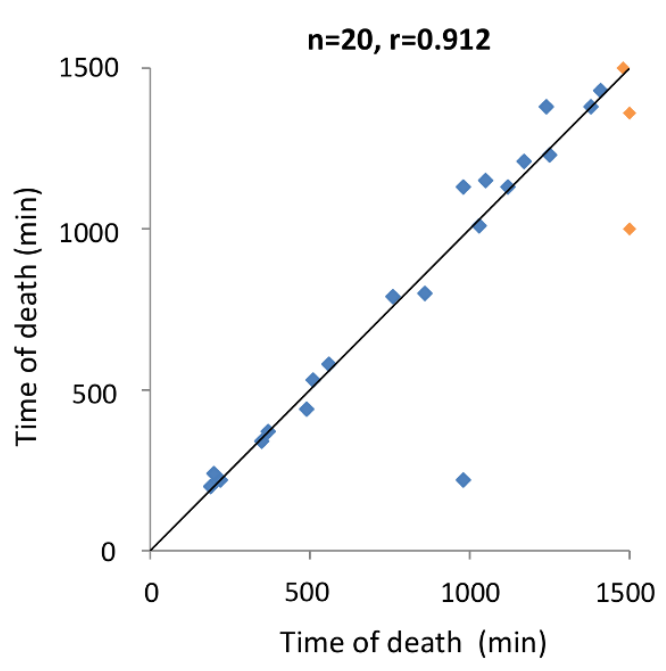

(b)

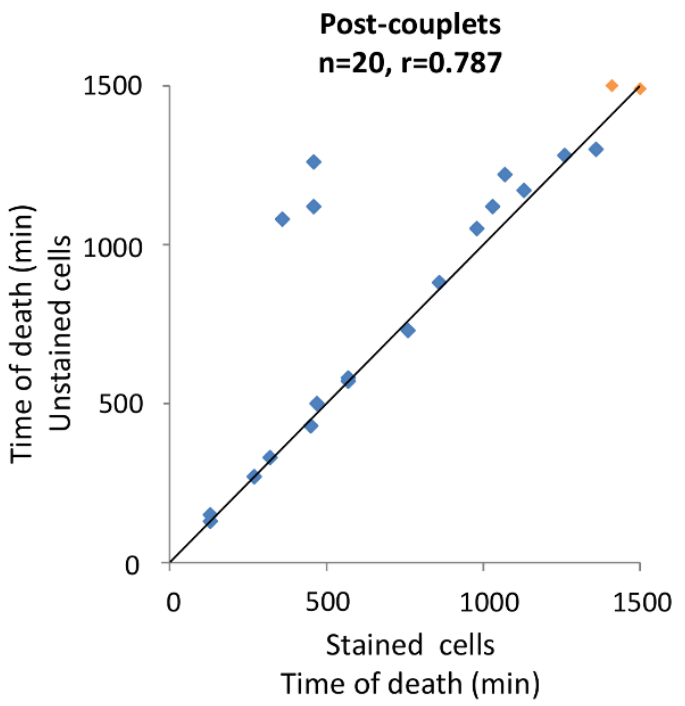

Figure $2 \mid$ APAP causes synchronized cell death in hepatocyte post-couplets. Primary cultured hepatocytes were stained using a CellTrace ${ }^{\mathrm{TM}}$ violet cell proliferation kit. After washing, unstained hepatocytes were added to the same dish. Cells were exposed to $10 \mathrm{mM}$ APAP and images were obtained every $10 \mathrm{~min}$ until 1,500 min by time-lapse microscopy. The time of death of each hepatocyte in the couplets was plotted as described in Fig. 1. (a) Couplets in which both hepatocytes were stained or unstained. (b) Couplets formed by an unstained hepatocyte and a stained hepatocyte (post-couplets).

(a)

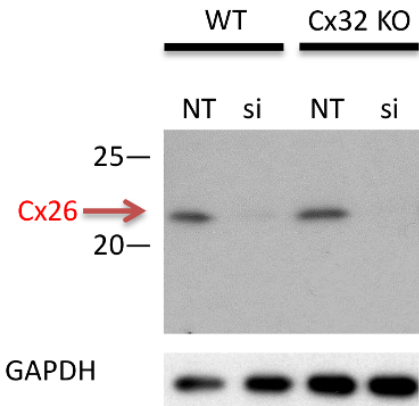

(b)

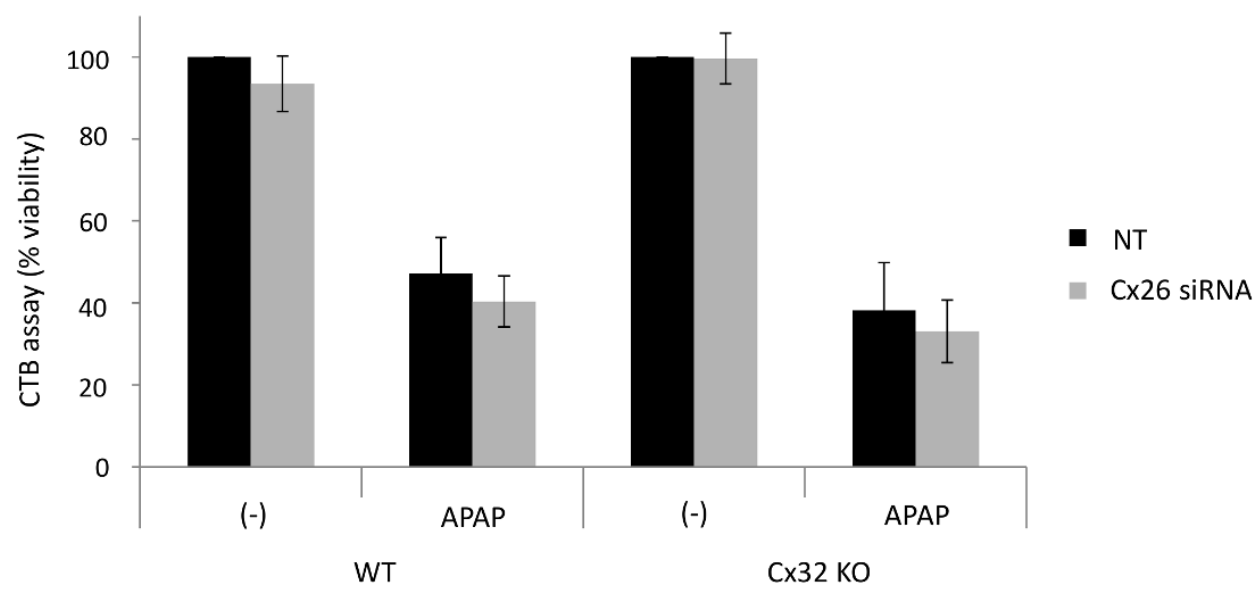

Figure 3 Knockdown of Cx26 expression by siRNA and no effect of Cx26 and Cx32 depletion on total hepatocyte viability after treatment with 10 mM APAP. (a) Hepatocytes prepared from WT and Cx32 KO mice were incubated with $100 \mathrm{nM}$ control (NT) siRNA or Cx26 siRNA (si) for 13 h. Then Cx26 and GAPDH were detected by western blotting. Full-length blots are presented in Supplementary Figure 6a b. (b) Viability of the indicated hepatocytes was measured by the CellTiter-Blue ${ }^{\mathrm{TM}}$ Cell Viability (CTB) assay with or without exposure to $10 \mathrm{mM}$ APAP for $24 \mathrm{~h}$. The viability of hepatocytes treated with control (NT) siRNA and not treated with APAP was set as $100 \%$. 
(a)
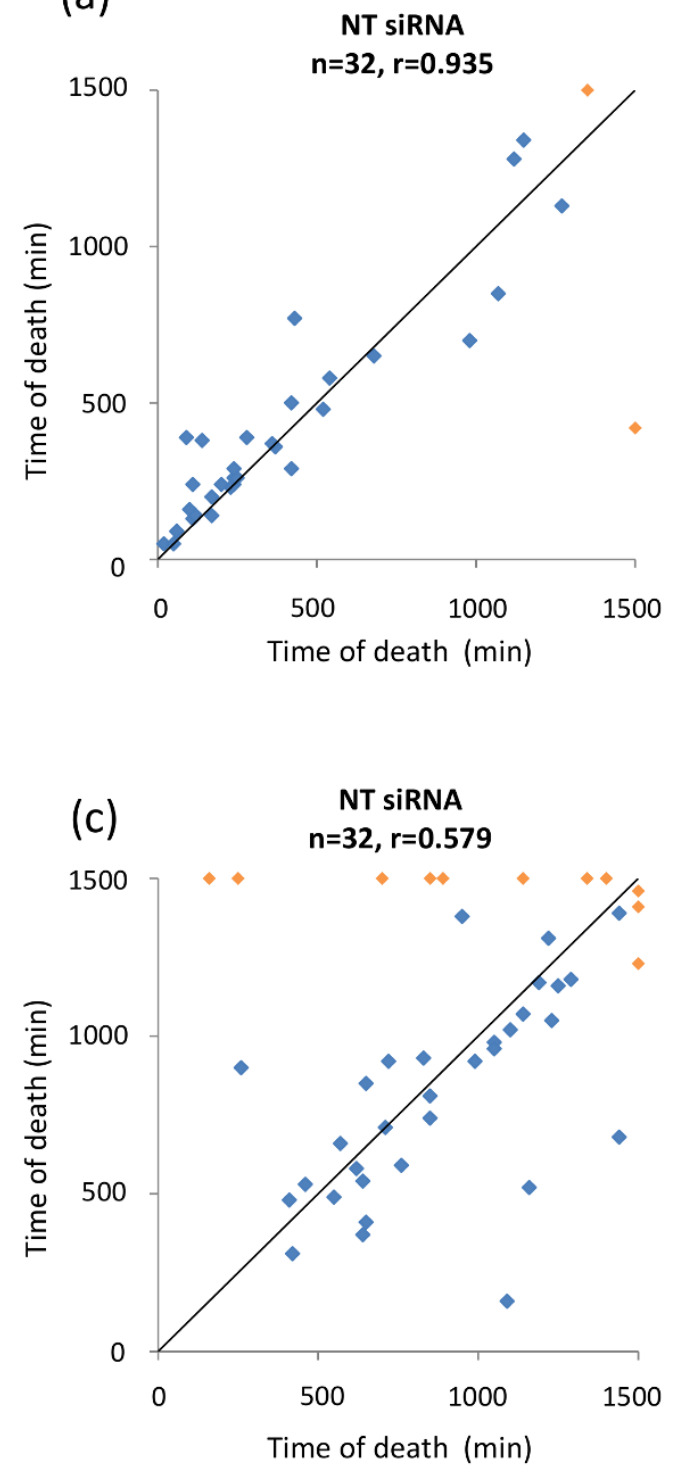

(b)
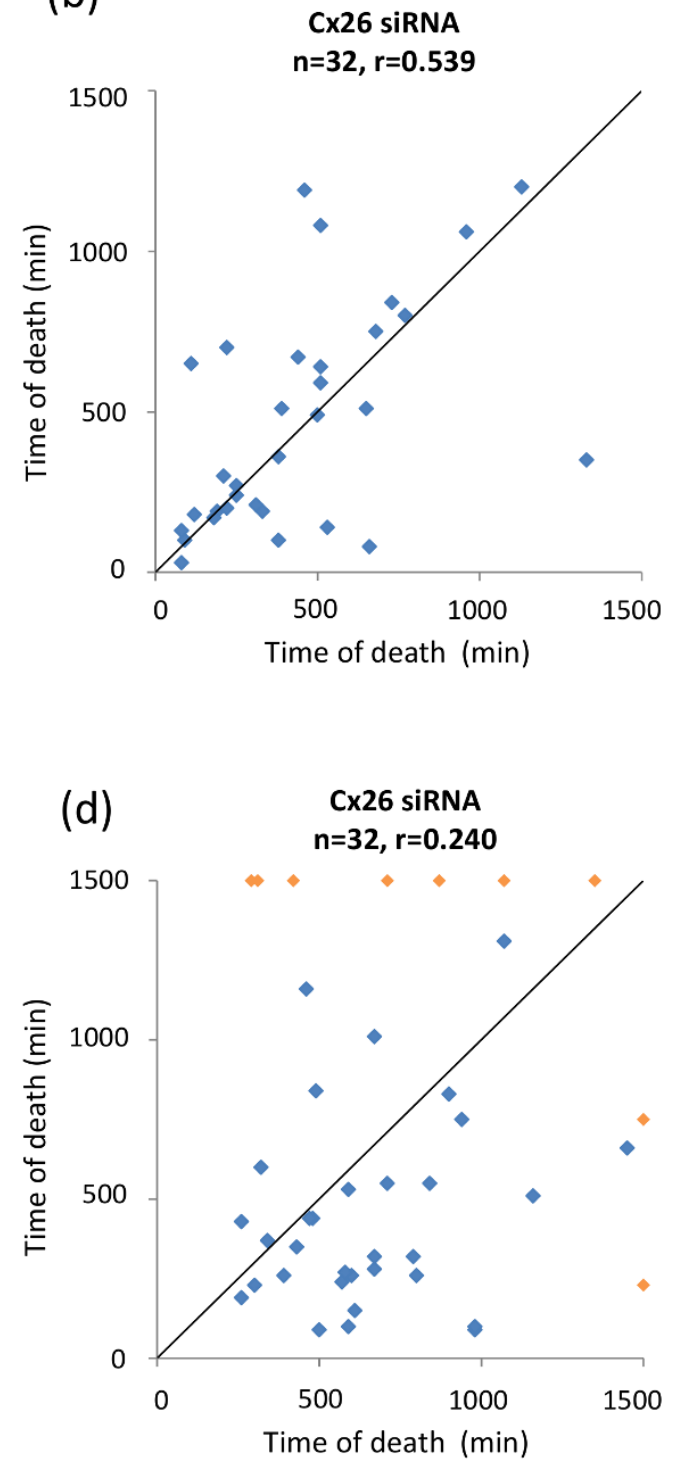

Figure 4 Synchronization of cell death after exposure to APAP is mediated by gap junctions. Hepatocytes were obtained from WT mice (a, b) or from Cx32 KO mice (c, d). These hepatocytes were incubated with either $100 \mathrm{nM}$ control (NT) siRNA (a, c) or Cx26 siRNA (b, d) for 13 h. Images were obtained every $10 \mathrm{~min}$ by time-lapse microscopy until 1,500 min after the start of exposure to $10 \mathrm{mM}$ APAP. The time of death of hepatocytes in couplets was measured and plotted as described in Fig. 1.

We also demonstrated that synchronization of cell death was mediated by cell-to-cell communication via gap junctions formed by $\mathrm{Cx} 26$ and $\mathrm{Cx} 32$.

Connexins not only form gap junctions between attached cells as a keystone of intercellular communication, but also form hemichannels in the cell membrane that contribute to paracrine signaling. Although we could not formally exclude the possibility of a role of connexin hemichannels in the synchronized death of attached hepatocytes induced by APAP, it seems unlikely because we observed that unattached hepatocytes located very close to other hepatocytes died independently of their neighboring cells (data not shown). Thus, intercellular gap junctions most likely play a crucial role in APAPinduced synchronized death of attached hepatocytes. To further investigate the role of cell-to-cell communication via gap junctions in APAP-induced cell death, we studied couplets formed by male and female hepatocytes that showed different sensitivity to APAP. We found that APAP-sensitive male hepatocytes were protected from death by attached APAP-resistant female hepatocytes in a manner that depended on functional gap junctions (Fig. 5).
The gap junction-dependent regulation of hepatocyte death could be explained by equal distribution of either death signaling molecules or death-suppressing molecules between attached hepatocytes. There have been reports that "danger signals" can be transferred and amplified to neighboring cells via gap junction-mediated intercellular communication ${ }^{15-17}$. NAPQI, a toxic byproduct of APAP metabolism, or more downstream effectors might pass through gap junctions to be distributed equally to attached hepatocytes ${ }^{18}$ and cause synchronized death. In the case of male-female couplets, more death signaling molecules are expected to be generated in male hepatocytes than in female hepatocytes and then are diluted in the two cells to a level below the threshold for inducing death via gap junctions, resulting in survival of both cells. Alternatively, intracellular "rescue signals" might be distributed equally to pairs of attached hepatocytes via gap junctions ${ }^{19,20}$, leading to synchronized death or to the protection of APAP-sensitive cells through transfer of a sufficient amount of death-suppressing molecules from attached APAP-resistant cells. Given that APAP-induced death involves reactive oxygen species (ROS), one candidate for such anti-death molecules is 
(a)

WT male hepatocytes Cx32 KO male hepatocytes
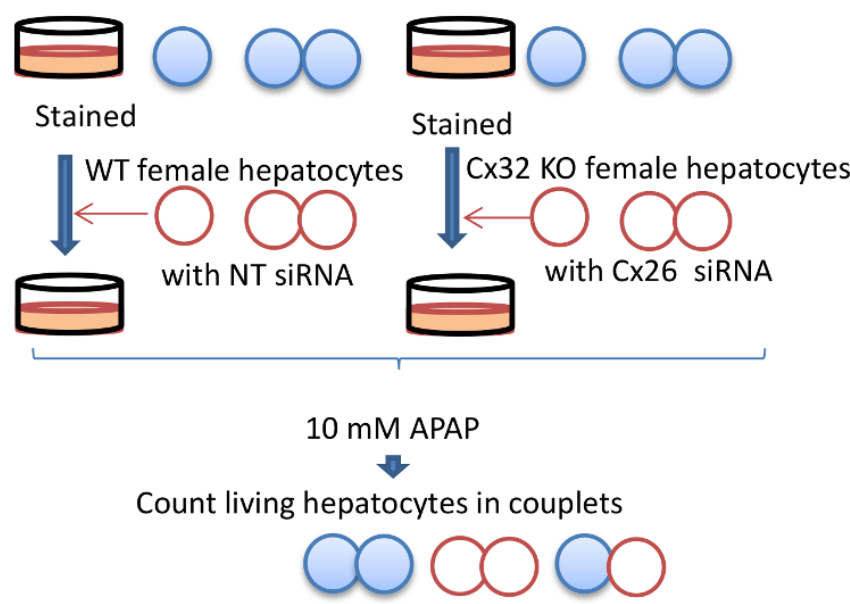

(c)

CX32 KO

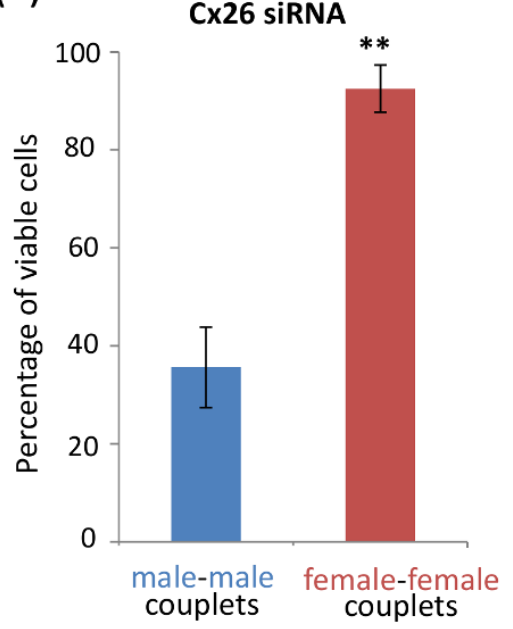

(b)

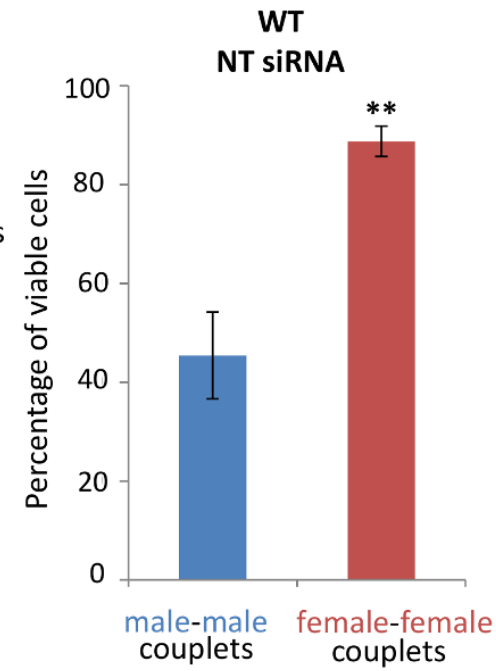

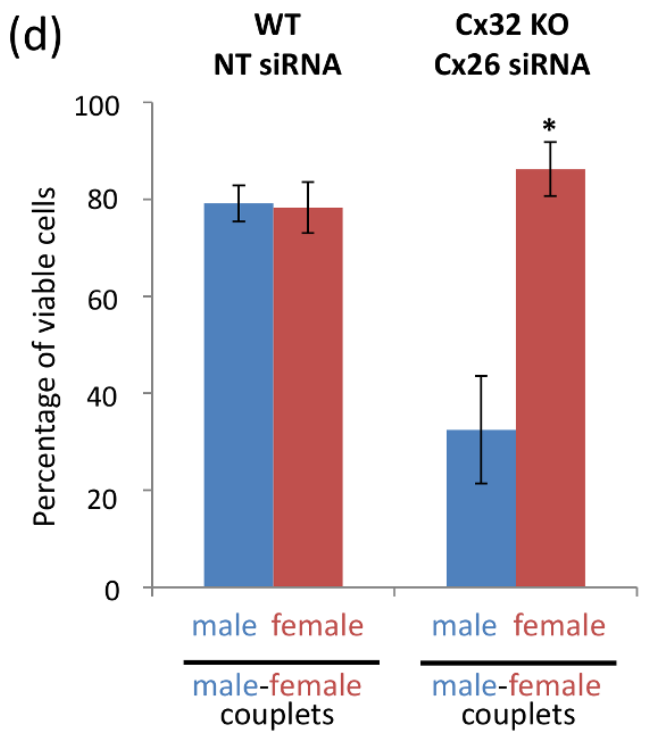

Figure 5 APAP-sensitive male hepatocytes were protected from APAP-induced death by APAP-resistant female hepatocytes via gap junctionmediated cell-to-cell communication. (a) Experimental design. Hepatocytes from male WT mice and male Cx32 KO mice were plated into dishes and stained using a CellTrace ${ }^{\mathrm{TM}}$ violet cell proliferation kit. After washing, unstained hepatocytes from female WT mice were added to the dishes together with control (NT) siRNA or hepatocytes from female Cx32 KO mice were added with Cx26 siRNA. After $13 \mathrm{~h}, 10 \mathrm{mM}$ APAP was added to each dish. (b, c, d) At 1,000 min after addition of APAP, the number of viable male and female cells was counted in 20 each of randomly selected couplets formed by malemale, female-female, or male-female hepatocytes. WT hepatocytes with NT siRNA (b, d) and Cx32 KO hepatocytes with Cx26 siRNA (c, d). In (d), blue and red bars respectively show the percentage of viable male and female cells in 20 male-female couplets. In (b-d), experiments were repeated $4-7$ times. ${ }^{* *} \mathrm{P}<0.001$ versus male-male couplets. ${ }^{*} \mathrm{P}<0.01$ versus male cells in male-female couplets from Cx32 KO hepatocytes treated with Cx26 siRNA.

$\mathrm{GSH}^{9,19}$. We indeed observed that (1) GSH levels were heterogeneous among hepatocytes, (2) GSH levels were similar between attached cells with functional gap junctions, but differed between attached hepatocytes that were deficient in gap junctions, and (3) GSH levels were generally higher in female hepatocytes than in male hepatocytes (Supplementary Fig. S5). These observations could explain some of our findings concerning APAP-induced death. First, the variation in the timing of death among groups of attached hepatocytes could be explained by heterogeneity of GSH levels among couplets. Differing GSH levels of cultured hepatocytes might be inherited from the in vivo setting, because it is well known that hepatocytes from the centrilobular area have a lower GSH content than hepatocytes from the periportal area. Second, we found that hepatocytes in couplets with functional gap junctions died in a synchronized manner, which could be explained by gap junction-dependent equal distribution of GSH in these couplets. Third, we discovered that male hepatocytes were protected from APAP-induced death by attachment to female hepatocytes, which could be explained by the higher GSH levels in many female hepatocytes. However, we are unable to exclude the possible involvement of other molecules in the synchronized death of attached hepatocytes.

In conclusion, sharing intracellular components via gap junctionmediated cell-to-cell communication could result in synchronized death or else lead to protection against toxic stimuli by equal distribution of death or survival molecules. Our findings raise the possibility that healthy cells could be a source of survival factors for 
injured cells via gap junctions, providing insight into a potential therapeutic strategy for patients with liver dysfunction and who might otherwise need liver transplantation.

\section{Methods}

Animals. Primary hepatocytes were isolated from male or female C57BL/6J mice (837 weeks old), which were obtained from Japan SLC (Shizuoka, Japan). Cx32heterozygous mice on a C57BL/6J background ${ }^{21}$ were kindly provided by Dr. Takayuki Okamoto (Mie University, Mie, Japan) and Dr. Hiroshi Yamasaki (Kwansei Gakuin University, Hyogo, Japan). The experimental protocol was approved by the Ethical Review Committee for Animal Experimentation of Osaka University Medical School.

Antibodies and chemicals. Anti-GAPDH and anti-connexin 26 antibodies were obtained from Merck Millipore (Billerica, MA). Celltrace ${ }^{\mathrm{TM}}$ violet cell proliferation kits and tetramethylrhodamine methyl ester (TMRM) were purchased from Life Technologies (Carlsbad, CA USA). Acetaminophen was obtained from Sigma (Tokyo, Japan), anti-Fas antibody was from BD Biosciences (Tokyo, Japan), and cycloheximide (CHX) was purchased from Wako (Osaka, Japan).

Hepatocyte isolation and culture. Murine hepatocytes were isolated by collagenase digestion, as described previously $y^{22}$. The viability of the isolated hepatocytes was routinely $90-95 \%$, as determined by the trypan blue exclusion assay. Hepatocytes were plated at a density of $2.5-8 \times 10^{4}$ cells in type I collagen-coated 12 -well dishes (Iwaki, Osaka, Japan) or $35 \mathrm{~mm}$ high $\mu$-Dishes (Nippon Genetics Co., Ltd., Tokyo, Japan). The cells were cultured in Leibovitz L-15 medium (Sigma, Tokyo, Japan) supplemented with $4 \mathrm{mM} \mathrm{L}$-glutamine (Sigma), antifungal and antibiotic agents (Nacalai Tesque, Kyoto, Japan), and 10\% v/v fetal bovine serum (FBS).

Western blotting. Proteins were extracted from hepatocytes by using lysis buffer (0.0625 M Tris-HCl, 5\% 2-mercaptoethanol, 2\% SDS, 5\% sucrose, 0.002\% bromophenol blue), separated by electrophoresis on $15 \%$ sodium dodecyl sulfatepolyacrylamide gels, and transferred to polyvinylidene fluoride (PVDF) membranes. The membranes were incubated with anti-GAPDH antibody $(1,5000)$ or anti-Cx26 antibody $(1: 670)$ with $5 \%$ skim milk in phosphate-buffered saline (PBS) containing $0.05 \% \mathrm{v} / \mathrm{v}$ Tween 20 , and proteins were detected by ECL prime (GE Healthcare Japan, Tokyo, Japan) using the appropriate horseradish peroxidase (HRP)-conjugated secondary antibodies.

Time-lapse microscopy. To detect APAP-induced cell death, the mitochondrial membrane potential was monitored by using TMRM $(0.25 \% \mathrm{v} / \mathrm{v})$ (Life Technologies, Carlsbad, CA USA) and a time-lapse fluorescence microscope (Fluoview FV10i, Olympus, Tokyo, Japan), and images were obtained every $10 \mathrm{~min}$. Loss of TMRM fluorescence was used as the indicator of cell death after exposure to $10 \mathrm{mM}$ APAP since most hepatocytes became PI-positive within $10 \mathrm{~min}$ of TMRM depletion. Prism 6 (GraphPad Software, Inc. La Jolla, CA USA) was used for analysis of the data.

Cell viability assay. In some experiments, cell viability was assessed with The Cell Titer Blue (CTB) assay reagent (Promega, Tokyo, Japan).

Cx26 siRNA. siRNA for Cx26 and the non-targeting control were obtained from Bonac Corporation (Fukuoka, Japan): Cx26 siRNA was 5'-

GGAAGGUGCCACUGAGAAA-3' and the non-targeting control was $5^{\prime}$ UACUAUUCGACACGCGAAG-3'. Transfection of siRNA into hepatocytes was performed with DharmaFECT1 (Thermo Fisher Scientific, Kanagawa, Japan) according to the supplier's protocol. After $13 \mathrm{~h}$ of siRNA treatment, hepatocytes were exposed to $10 \mathrm{mM}$ APAP.

Genotyping. Tail DNA from WT and Cx32 KO mice was subjected to genotyping using three primers simultaneously. The WT allele for Cx32 was detected using the following pair of oligonucleotides: $5^{\prime}$-CCATAAGTCAGGTGTAAAGGAGC-3' (primer 1) and 5'- GAGCATAAAGACAGTGAAGACGG-3' (primer 2). For the Cx32 KO allele, primer 1 was used with $5^{\prime}$-ATCATGCGAAACGATCCTTCATCC$3^{\prime}$ (primer 3), which is specific for the neo cassette. The Neo cassette was inserted into exon 2 of the $\mathrm{Cx} 32$ gene. PCR with these primers amplified a DNA fragment of $888 \mathrm{bp}$ for WT mice and $414 \mathrm{bp}$ for Cx32 KO mice ${ }^{23}$.

Statistical analysis. Results were shown as the mean \pm SD. Comparison between two groups was performed with a two-tailed Student's t-test using Excel software.

1. Ryoo, H. D., Gorenc, T. \& Steller, H. Apoptotic cells can induce compensatory cell proliferation through the JNK and the Wingless signaling pathways. Developmental cell 7, 491-501, doi:10.1016/j.devcel.2004.08.019 (2004).

2. Klee, P. et al. Connexins protect mouse pancreatic beta cells against apoptosis. J Clin Invest 121, 4870-4879, doi:10.1172/jci40509 (2011).

3. Asamoto, M., Hokaiwado, N., Murasaki, T. \& Shirai, T. Connexin 32 dominantnegative mutant transgenic rats are resistant to hepatic damage by chemicals. Hepatology 40, 205-210, doi:10.1002/hep.20256 (2004).
4. Naiki-Ito, A. et al. Gap junction dysfunction reduces acetaminophen hepatotoxicity with impact on apoptotic signaling and connexin 43 protein induction in rat. Toxicol Pathol 38, 280-286, doi:10.1177/0192623309357951 (2010).

5. Vinken, M. et al. Connexins and their channels in cell growth and cell death. Cell Signal 18, 592-600, doi:10.1016/j.cellsig.2005.08.012 (2006).

6. Feine, I., Pinkas, I., Salomon, Y. \& Scherz, A. Local oxidative stress expansion through endothelial cells--a key role for gap junction intercellular communication. PLoS One 7, e41633, doi:10.1371/journal.pone.0041633 (2012).

7. Decrock, E. et al. Connexin-related signaling in cell death: to live or let die? Cell Death Differ 16, 524-536, doi:10.1038/cdd.2008.196 (2009).

8. Eugenin, E. A. et al. The role of gap junction channels during physiologic and pathologic conditions of the human central nervous system. J Neuroimmune Pharmacol 7, 499-518, doi:10.1007/s11481-012-9352-5 (2012).

9. Saito, C., Zwingmann, C. \& Jaeschke, H. Novel mechanisms of protection against acetaminophen hepatotoxicity in mice by glutathione and $\mathrm{N}$-acetylcysteine. Hepatology 51, 246-254, doi:10.1002/hep.23267 (2010).

10. Badr, M. Z., Belinsky, S. A., Kauffman, F. C. \& Thurman, R. G. Mechanism of hepatotoxicity to periportal regions of the liver lobule due to allyl alcohol: role of oxygen and lipid peroxidation. J Pharmacol Exp Ther 238, 1138-1142 (1986).

11. Gabriel, H. D. et al. Transplacental uptake of glucose is decreased in embryonic lethal connexin26-deficient mice. J Cell Biol 140, 1453-1461 (1998).

12. McConnachie, L. A. et al. Glutamate cysteine ligase modifier subunit deficiency and gender as determinants of acetaminophen-induced hepatotoxicity in mice. Toxicol Sci 99, 628-636, doi:10.1093/toxsci/kfm165 (2007).

13. Dai, G., He, L., Chou, N. \& Wan, Y. J. Acetaminophen metabolism does not contribute to gender difference in its hepatotoxicity in mouse. Toxicol Sci 92, 33-41, doi:10.1093/toxsci/kfj192 (2006).

14. Masubuchi, Y., Nakayama, J. \& Watanabe, Y. Sex difference in susceptibility to acetaminophen hepatotoxicity is reversed by buthionine sulfoximine. Toxicology 287, 54-60, doi:10.1016/j.tox.2011.05.018 (2011).

15. Lin, J. H. et al. Gap-junction-mediated propagation and amplification of cell injury. Nat Neurosci 1, 494-500, doi:10.1038/2210 (1998).

16. Kasper, C. A. et al. Cell-cell propagation of NF-kappaB transcription factor and MAP kinase activation amplifies innate immunity against bacterial infection. Immunity 33, 804-816, doi:10.1016/j.immuni.2010.10.015 (2010).

17. Krysko, D. V., Leybaert, L., Vandenabeele, P. \& D'Herde, K. Gap junctions and the propagation of cell survival and cell death signals. Apoptosis 10, 459-469, doi:10.1007/s10495-005-1875-2 (2005).

18. Patel, S. J. et al. Gap junction inhibition prevents drug-induced liver toxicity and fulminant hepatic failure. Nat Biotechnol 30, 179-183, doi:10.1038/nbt.2089 (2012).

19. Igarashi, I. et al. Role of connexin 32 in acetaminophen toxicity in a knockout mice model. Exp Toxicol Pathol, doi:10.1016/j.etp.2013.10.002 (2013).

20. Contreras, J. E. et al. Role of connexin-based gap junction channels and hemichannels in ischemia-induced cell death in nervous tissue. Brain Res Brain Res Rev 47, 290-303, doi:10.1016/j.brainresrev.2004.08.002 (2004).

21. Nelles, E. et al. Defective propagation of signals generated by sympathetic nerve stimulation in the liver of connexin32-deficient mice. Proc Natl Acad Sci U S A 93, 9565-9570 (1996).

22. Shinzawa, K., Watanabe, Y. \& Akaike, T. Primary cultured murine hepatocytes but not hepatoma cells regulate the cell number through density-dependent cell death. Cell Death Differ 2, 133-140 (1995).

23. Temme, A., Ott, T., Dombrowski, F. \& Willecke, K. The extent of synchronous initiation and termination of DNA synthesis in regenerating mouse liver is dependent on connexin32 expressing gap junctions. J Hepatol 32, 627-635 (2000).

\section{Acknowledgments}

We are grateful to Drs. Takayuki Okamoto (Mie University, Mie, Japan) and Hiroshi Yamasaki (Kwansei Gakuin University, Hyogo, Japan) for providing the Cx32 KO mice. We also thank Dr. Takashi Kodama (Osaka University, Osaka, Japan) for giving us advice about statistical analysis. This work was supported by the Japan Society for the Promotion of Science, a Grant-in-Aid for Creative Scientific Research, and the Global COE program of the Ministry of Education, Culture, Sports, Science and Technology of Japan.

\section{Author contributions}

C.S., K.S. and Y.T. designed the experiments and C.S. performed the experiments. C.S., K.S. and Y.T. wrote the main manuscript text and C.S. prepared all figures. All authors reviewed the manuscript.

\section{Additional information}

Supplementary information accompanies this paper at http://www.nature.com/ scientificreports

Competing financial interests: The authors declare no competing financial interests. How to cite this article: Saito, C., Shinzawa, K. \& Tsujimoto, Y. Synchronized necrotic death of attached hepatocytes mediated via gap junctions. Sci. Rep. 4, 5169; DOI:10.1038/ srep05169 (2014). 
(i) This work is licensed under a Creative Commons Attribution-NonCommercialNoDerivs 3.0 Unported License. The images in this article are included in the article's Creative Commons license, unless indicated otherwise in the image credit; if the image is not included under the Creative Commons license, users will need to obtain permission from the license holder in order to reproduce the image. To view a copy of this license, visit http://creativecommons.org/licenses/by-nc-nd/3.0/ 